\title{
CATÁLISE ASSIMÉTRICA NO BRASIL: DESENVOLVIMENTO E POTENCIALIDADES PARA O AVANÇO DA INDÚSTRIA QUÍMICA BRASILEIRA
}

\author{
Antonio Luiz Braga* \\ Departamento de Química, Universidade Federal de Santa Catarina, 88040-900 Florianópolis - SC, Brasil \\ Diogo Seibert Lüdtke e Paulo Henrique Schneider \\ Instituto de Química, Universidade Federal do Rio Grande do Sul, 91501-970 Porto Alegre - RS, Brasil \\ Leandro Helgueira Andrade \\ Instituto de Química, Universidade de São Paulo, 05508-900 São Paulo - SP, Brasil \\ Márcio Weber Paixão \\ Departamento de Química, Universidade Federal de São Carlos, 13565-905 São Carlos - SP, Brasil
}

Recebido em 11/7/13; aceito em 9/10/13; publicado na web em 24/10/13

\begin{abstract}
ASYMMETRIC CATALYSIS IN BRAZIL: DEVELOPMENT AND POTENTIAL FOR ADVANCEMENT OF BRAZILIAN CHEMICAL INDUSTRY. The preparation of enantiomerically pure or enriched substances is of fundamental importance to pharmaceutical, food, agrochemical, and cosmetics industries and involves a growing market of hundreds of billions of dollars. However, most chemical processes used for their production are not environmentally friendly because in most cases, stoichiometric amounts of chiral inductors are used and substantial waste is produced. In this context, asymmetric catalysis has emerged as an efficient tool for the synthesis of enantiomerically enriched compounds using chiral catalysts. More specifically, considering the current scenario in the Brazilian chemical industry, especially that of pharmaceuticals, the immediate prospect for the use of synthetic routes developed in Brazil in an enantioselective fashion or even the discovery of new drugs is practically null. Currently, the industrial production of drugs in Brazil is primarily focused on the production of generic drugs and is basically supported by imports of intermediates from China and India. In order to change this panorama and move forward toward the gradual incorporation of genuinely Brazilian synthetic routes, strong incentive policies, especially those related to continuous funding, will be needed. These incentives could be a breakthrough once we establish several research groups working in the area of organic synthesis and on the development and application of chiral organocatalysts and ligands in asymmetric catalysis, thus contributing to boost the development of the Brazilian chemical industry. Considering these circumstances, Brazil can benefit from this opportunity because we have a wide biodiversity and a large pool of natural resources that can be used as starting materials for the production of new chiral catalysts and are creating competence in asymmetric catalysis and related areas. This may decisively contribute to the growth of chemistry in our country.
\end{abstract}

Keywords: asymmetric catalysis; chiral compounds; chiral catalyst.

\section{INTRODUÇÃO}

Com o objetivo de fomentar e contribuir na formulação de novas políticas de C\&T, especialmente para a área de Química, apresentam-se, nesse artigo, diagnósticos, reflexões e discussões sobre a atual situação da área de Catálise Assimétrica no Brasil. Propõem-se, ainda, ações que permitam à Química do País contribuir para um futuro próspero, seguro e sustentável para sua população. A presente proposta está de acordo com os position papers "Química sem Fronteiras" da Química Nova, ${ }^{1}$ nos temas centrais: Indústria Química e Inovação, nos setores farmoquímicos, agroquímicos e de alimentos. Enquadra-se, também, nos temas transversais, nos setores de inovação e agregação de valores.

Durante as últimas décadas grandes esforços vêm sendo realizados no sentido de se estabelecer rotas enantiosseletivas para a preparação de substâncias enantiomericamente puras ou enriquecidas, devido à sua importância em indústrias farmoquímicas, de alimentos, na agricultura e inclusive na área de novos materiais, tais como polímeros e cristais líquidos quirais. ${ }^{2}$ No campo farmoquímico, a importância de se obter compostos quirais não racêmicos está vinculada entre a atividade biológica e o estereoisomerismo, já que a maioria dos receptores biológicos são sistemas protéicos,

*e-mail: braga.antonio@ufsc.br conferindo aos organismos vivos a característica de um ambiente quiral. Em muitos casos, a atividade farmacológica está restrita a um dos enantiômeros enquanto que o outro pode ser responsável por reações adversas e/ou contaminação ambiental. ${ }^{3}$ Devido à atividade farmacológica distinta, os enantiômeros de fármacos quirais podem ainda diferir em sua toxicidade. Da mesma forma, a distribuição dos diferentes enantiômeros do mesmo fármaco quiral no ambiente aquático e biota também pode ser estereosseletiva, e com isso a sua degradação durante o tratamento de águas residuais e no ambiente pode ocorrer de maneira estereosseletiva e conduzir a produtos quirais de toxicidades variadas, podendo levar a uma contaminação significativa do meio ambiente.

Além da importância biológica, deve-se destacar, também, que a comercialização de compostos enatiomericamente puros para fins farmacêuticos movimentam cifras significativas. Somente no ano de 2002 as vendas atingiram mais de US\$ 159 bilhões, ${ }^{4}$ estando hoje acima de US\$ 200 bilhões. ${ }^{5}$ Desse total, a comercialização de pequenas moléculas representam cerca de dois terços e biofármacos apenas um terço. Ao mesmo tempo, o FDA, o principal órgão regulador de medicamentos, alimentos e cosméticos dos Estados Unidos tem se tornado cada vez mais relutante em permitir a introdução de novos fármacos racêmicos, pelas razões anteriormente mencionadas.

A maioria das rotas sintéticas ainda utilizadas para a síntese de matérias-primas ou de pequenas moléculas quirais não envolvem 
métodos catalíticos enantiosseletivos. Normalmente são utilizadas metodologias que envolvem auxiliares quirais, separação por cromatografia quiral ou resolução de misturas racêmicas com um agente opticamente puro. No entanto, esses processos não são economicamente e ambientalmente adequados, pois envolvem reações químicas adicionais, utilizam quantidades estequiométricas de indutores quirais e ainda geram uma quantidade significativa de resíduos, que necessitam de tratamento adequado, agregando custos aos processos. Nos últimos anos, a preocupação com estes inconvenientes vem aumentando, havendo uma pressão crescente sobre as indústrias químicas, tanto por parte da sociedade civil, como das autoridades governamentais, no sentido de aprimorar o desenvolvimento de processos que sejam cada vez menos prejudiciais ao meio ambiente. ${ }^{6}$ Como consequência, diversas oportunidades em química fina na preparação de intermediários enantiomericamente puros ou enriquecidos por métodos mais sustentáveis deverão surgir, podendo mudar o mapa atual de produção global.

Neste contexto, a catálise assimétrica surge como um processo fundamental, eficiente e seletivo para a síntese de pequenas moléculas enantiomericamente puras ou enriquecidas a partir de moléculas aquirais utilizando um catalisador quiral. ${ }^{7}$

Considerando-se o atual cenário apresentado pela indústria química brasileira, especialmente aquele vinculado ao setor farmacêutico, as perspectivas imediatas de utilização de rotas sintéticas, principalmente enantiosseletivas para a produção ou até mesmo para a descoberta de novos fármacos é praticamente nula. A indústria de produção de fármacos no Brasil tem se concentrado, sobretudo, na produção de genéricos e é apoiada majoritariamente em importações da China e da Índia. Para uma mudança nesse cenário e incorporação gradual de rotas sintéticas genuinamente brasileiras, serão necessárias políticas de incentivo (financiamento) e fortalecimento de pesquisas em síntese orgânica, oriundos tanto das agências de fomento governamentais e parcerias com o setor produtivo. A atual situação somente começará a ser revertida com forte apoio das agências de fomento a projetos de pesquisa em química orgânica sintética, dentre os quais se inclui a área de catálise assimétrica. O Brasil poderá facilmente se engajar nesta corrente, uma vez que vem criando competência nessa área e hoje conta com diversos grupos de pesquisas atuando no desenvolvimento e aplicação de ligantes e organocatalisadores quirais em catálise assimétrica, como será abordado mais adiante.

Os processos catalíticos enantiosseletivos podem ser agrupados em heterogêneos ou homogêneos, baseados na fase em que ocorre a reação. De modo geral, ainda pode-se dividir a catálise assimétrica em três sub-áreas: organometálica, enzimática e organocatálise, com base no tipo de catalisador empregado. Com o atual estágio de desenvolvimento da biotecnologia e de processos de imobilização de catalisadores homogêneos em suportes sólidos, pode-se predizer que, no futuro, estas diferenças serão progressivamente menos distinguidas, levando inclusive à crescente utilização de sistemas catalíticos mistos.

Nas últimas décadas vem se observando um interesse crescente no desenvolvimento e aplicação de catalisadores homogêneos quirais em processos sintéticos, fundamentalmente devido à maior seletividade conseguida nos produtos finais e também por permitirem aplicações industriais em grande escala.

Isso foi evidenciado em 2001 quando a Academia Real de Ciências da Suécia laureou os químicos William S. Knowles, K. Barry Sharpless e Ryoji Noyori, pelas suas notáveis contribuições no âmbito da síntese de novos catalisadores quirais homogêneos e suas aplicações em catálise assimétrica. ${ }^{8}$ Os estudos envolveram processos catalíticos enantiosseletivos de hidrogenação, hidroformilação, epoxidação e di-hidroxilação, permitindo a preparação de matérias-primas e moléculas enantiomericamente puras ou enriquecidas em escala industrial.
Estas descobertas estiveram na origem de inúmeros processos de síntese patenteados e com aplicação industrial, com impacto imediato na área de fármacos, agroquímicos, alimentos, perfumaria, saponificantes, apenas para citar algumas. O exemplo mais significativo da época envolveu a produção em escala industrial do fármaco conhecido como ( $S$ )-DOPA (3,4-di-hidroxifenilalanina), utilizado no tratamento de Parkinson, e que foi patenteado pela Empresa Monsanto. Knowles liderou o desenvolvimento deste projeto, onde o aminoácido desejado foi obtido com elevados excessos enantioméricos $(e e)$, por meio de uma reação catalítica assimétrica de hidrogenação de um alceno utilizando como ligante uma fosfina quiral, $(R, R)$-DIPAMP (Esquema 1$){ }^{9}$

Da mesma forma, diversas classes de fármacos puderam ou ainda serão beneficiadas pelos avanços da catálise assimétrica. Dentre as já beneficiadas citam-se: anti-inflamatórios não esteroidais, beta-bloqueadores, agentes anti-hipertensivos, antibióticos, antidepressivos, anticonvulsivantes, antivirais, anti-hipercolesterolemicos, antiparkinsonianos, etc. No entanto, é válido ressaltar que, em sua ampla maioria, a utilização de métodos catalíticos e assimétricos para a produção de insumos enantiomericamente puros ou enriquecidos está centrada na utilização de reações de hidrogenação e oxidação. Ainda há uma enorme lacuna a ser preenchida no que diz respeito, especialmente, à formação estereocontrolada de ligações carbono-carbono. ${ }^{10}$

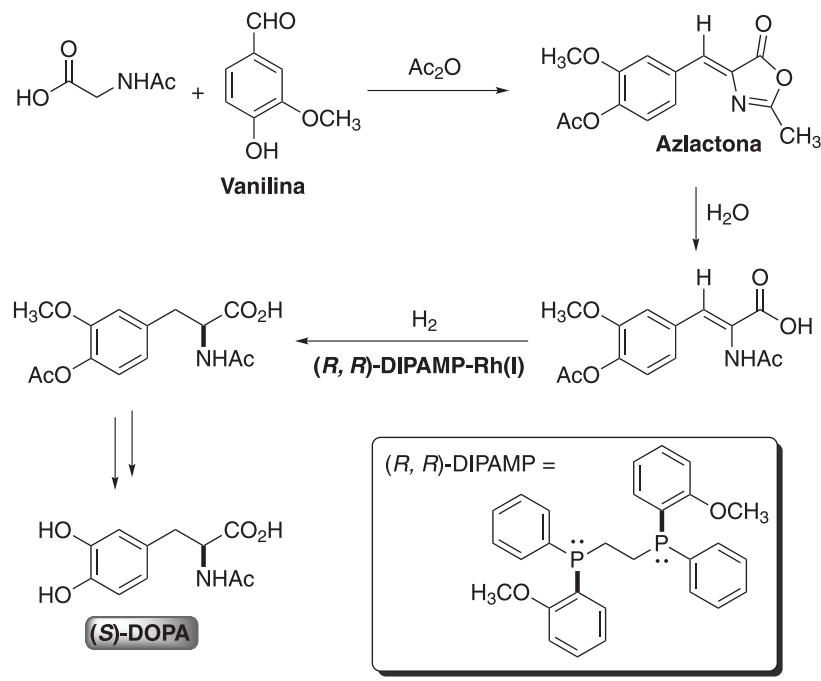

Esquema 1. Rota sintética patenteada da Monsanto para a síntese do (S)DOPA

As grandes contribuições desses pesquisadores foram além do impacto de suas descobertas, pois influenciaram diversos grupos de pesquisas com conceitos e princípios em catálise assimétrica e que vêm permitindo a o desenvolvimento de processos catalíticos industriais ambientalmente mais adequados.

\section{A CATÁLISE ASSIMÉTRICA NO BRASIL}

A catálise assimétrica no Brasil está baseada fundamentalmente em sistemas homogêneos, nos segmentos de biocatálise, organocatálise e catálise organometálica, salvo alguns exemplos de trabalhos pontuais em catálise heterogênea. ${ }^{11}$

Atualmente vários grupos vêm atuando no desenvolvimento e aplicação de catalisadores quirais/biocatalisadores em catálise assimétrica, colocando o Brasil em um cenário promissor, tanto na potencial aplicação industrial, quanto no avanço científico e geração de recursos humanos altamente especializados. A busca por catalisadores/biocatalisadores privilegiados, que podem ser aplicados de 
modo eficaz em diversas classes de reações enantiosseletivas, é uma atividade fundamental nesses processos. ${ }^{12}$

\section{Biocatálise}

O uso de enzimas como catalisadores de importantes reações químicas para a produção de pequenas moléculas quirais é um assunto que vem chamando atenção da comunidade científica e industrial. Em grande parte esse interesse está relacionado com várias características desses biocatalisadores, que se destacam por sua alta biodegradabilidade, seletividade (quimiosseletividade, estereosseletividade e regiosseletividade) e condições brandas das reações que participam, pois podem ser realizadas em sistemas aquosos e à temperatura e pressão ambiente.

A biocatálise tornou-se efetivamente importante quando pôde ser aplicada em escala industrial para a síntese de diversos intermediários químicos quirais com reconhecido valor comercial. ${ }^{13}$ Por exemplo, a síntese de antibióticos beta-lactâmicos envolve a preparação de um precursor ácido 6-aminopenicilânico (6-APA) a partir da hidrólise da penicilina $\mathrm{G}$ catalisada por penicilina $\mathrm{G}$ acilase (Esquema 2). ${ }^{14}$

No Brasil, o uso da biocatálise na síntese de compostos quirais foi uma das subáreas da catálise assimétrica que primeiro se estabeleceu, ${ }^{15}$ e hoje conta com diversos grupos de pesquisa consolidados ou em consolidação, baseados na Unicamp, ${ }^{15}$ UFSC, ${ }^{16}$ na USP-São Carlos,${ }^{17}$ na UFRJ $;{ }^{18}$ na UFC ${ }^{19-21}$ e no IQ-USP. ${ }^{22-24}$ Dentre outros estudos envolvendo enzimas isoladas ${ }^{23}$ ou micro-organismos, ${ }^{24} \mathrm{com}$ a síntese de diversas classes de moléculas quirais, foi desenvolvido recentemente um processo importante, que trata da oxidação enzimática de compostos de boro (Esquema 3). ${ }^{25}$

Nesse estudo explorou-se a quimiosseletividade e a enantiosseletividade de reações catalisadas por diversas Baeyer-Villiger monooxigenases (BVMO). Observou-se que nos substratos com duas funções orgânicas oxidáveis, a oxidação do boro catalisada por BVMO ocorreu preferencialmente em comparação com a reação de Baeyer-Villiger ou reação de epoxidação. Uma aplicação interessante desse protocolo foi realizada por meio de reações com substratos racêmicos de boro. Nesse caso, observou-se que as BVMO foram eficientes em catalisar a oxidação de boro de forma enantiosseletiva, o que resultou num processo de resolução cinética oxidativa. Outro aspecto importante relacionado à biocatálise é a busca por novas enzimas, provenientes de diferentes biomassas, para atuarem como biocatalisadores em diferentes reações. ${ }^{26} \mathrm{Em}$ estudos recentes envolvendo amostras de solo da Floresta Amazônica, por exemplo, isolou-se uma bactéria, Arthrobacteratrocyaneus produtora de álcool desidrogenase, que apresentou grande eficiência na oxidação enantiosseletiva de uma série de $(S)$-feniletanóis, resultando em um processo de resolução cinética oxidativa de misturas racêmicas desses álcoois. Nesta subárea, outros grupos de pesquisa deverão se estabelecer no Brasil, uma vez que existe uma grande diversidade de micro-organismos ainda desconhecidos e que poderão ser explorados no desenvolvimento de diversos processos de produção ambientalmente aceitáveis de moléculas quirais. Além disso, também existe uma lacuna nesse campo referente ao desenvolvimento de processos em larga escala envolvendo biocatalisadores. Adicionalmente, a crescente interação dessa área com a biologia molecular deverá contribuir decisivamente para o crescimento da catálise assimétrica e da química no país.

\section{Catálise organometálica}

A catálise organometálica assimétrica é um dos campos de estudo mais abrangentes do Brasil. Porém, poucos grupos efetivamente desenvolvem pesquisas focadas no desenvolvimento de novos catalisadores quirais, ${ }^{27}$ sendo que boa parte dos trabalhos desenvolvidos envolvem artigos de grupos pontuais, ${ }^{28}$ dentre os quais cabe destacar os avanços recentes que vêm sendo relatados pelo grupo do Prof. Correia no desenvolvimento da versão catalítica e assimétrica da reação de Heck-Matsuda de olefinas cíclicas e acíclicas. ${ }^{29}$

Um dos grupos mais consolidados nessa área utiliza como estratégia o desenvolvimento de catalisadores/ligantes provenientes de aminoácidos, que são compostos abundantes de origem natural. ${ }^{30}$ Um exemplo interessante envolve a síntese e aplicação do ligante quiral dissulfeto oxazolidínico na adição de dietilzinco em aldeídos resultando na produção de álcoois secundários quirais com ee de até $99 \%$ (Esquema 4$),{ }^{30}$

Observou-se que com o uso de apenas 2 mol \% do dissulfeto oxazolidínico obteve-se uma série de álcoois secundários em excelentes

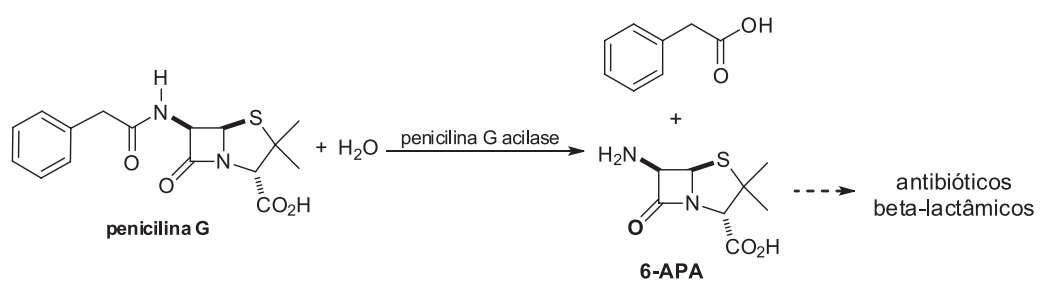

Esquema 2. Produção de ácido 6-aminopenicilânico (6-APA)

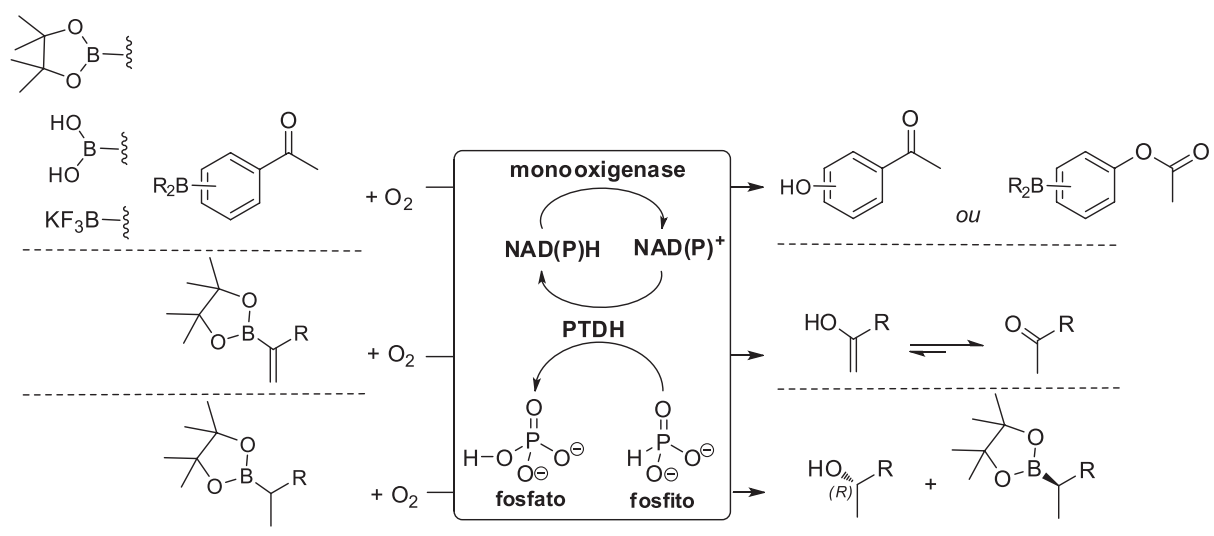

Esquema 3. Seletividade de reações de compostos de boro catalisadas por monooxigenases. $P T D H=$ Fosfito desidrogenase 


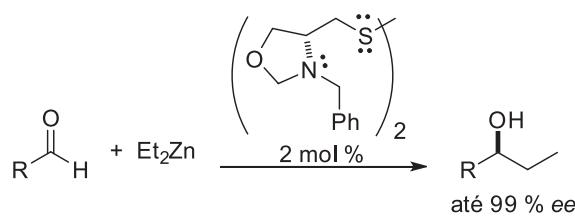

Esquema 4. Catalisador enantiopuro contendo enxofre para adição assimétrica de dietilzinco a aldeídos

rendimentos e ee. De acordo com Noyori, ${ }^{31} \mathrm{o}$ estado de transição dessa reação passa por espécies diastereiosoméricas dinucleares de zinco (Esquema 5).

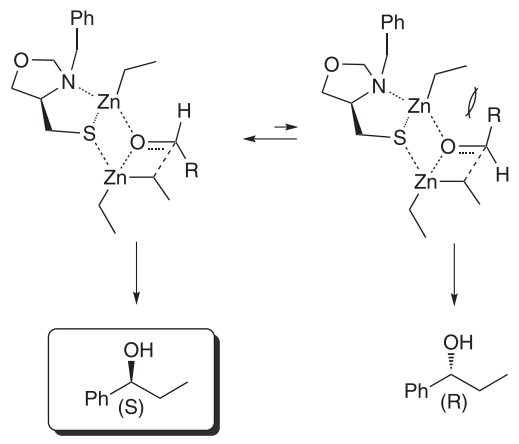

Esquema 5. possível estado de transição

O sucesso no desenvolvimento desse tipo de ligante/catalisador levou à adoção de uma estratégia sintética inspirada em química combinatória, para desenvolver diferentes classes de ligantes. ${ }^{30} \mathrm{~A}$ partir da combinação sistemática de blocos sintéticos, um grande número de ligantes/catalisadores puderam ser rapidamente preparados, em contraste com as dificuldades oriundas de um planejamento envolvendo reações clássicas sequenciais por uma estratégia linear.

Com isso, e a partir da escolha de blocos sintéticos apropriados, encontrou-se os melhores candidatos em um processo de seleção para diferentes tipos de reações enantiosseletivas clássicas. Além disto, a informação quiral veio de fontes naturais enantiomericamente puras, tais como os aminoácidos.

A base para uma rota combinatória é a utilização de um sistema apropriado na preparação de ligantes com as seguintes propriedades: 1. Blocos sintéticos baratos, prontamente aplicáveis e de fácil obtenção; 2. Ligantes enantiopuros; 3 . Síntese quantitativa e quimioseletiva; 4. Alta diversidade estrutural pela introdução de diferentes blocos de sintéticos (caráter modular).

Graficamente, o Esquema 6 poderia ser considerado para a síntese combinatória do ligante dissulfeto e outros análogos. Este catalisador contendo enxofre se enquadra perfeitamente em uma rota combinatória, uma vez que ele se adapta a todas as propriedades previamente descritas. A $(R)$-Cisteína seria a molécula base que contém a informação quiral. De maneira a aumentar ainda mais a diversidade estrutural, alguns outros aminoácidos naturais, tais como $(L)$-Homocisteína e a $(L)$-Penicilamina, ainda poderiam ser utilizados como blocos sintéticos (Figura 1).<smiles>NC(CS)C(=O)O</smiles>

(L)-Cisteína<smiles>NC(CCS)C(=O)O</smiles>

(L)-Homocisteína<smiles>CC(C)(S)C(N)C(=O)O</smiles>

(L)-Penicilamina
Figura 1. Blocos de construção com informação quiral

A título de exemplo, com somente 3 tipos de blocos sintéticos "I" e partindo-se de cada um dos 10 aldeídos e cetonas dos blocos (II e III) poderia se obter aritmeticamente 600 diferentes ligantes $(3 \times 10 \times 10 \times 2$ isômeros de posição dos blocos II e III). A alquilação do tiol ou a combinação deste (oxidação) levando a formação dos dissulfetos correspondentes, resultaria num aumento substancial do número de ligantes sintetizados, o que é uma estratégia interessante no refinamento de um ligante/catalisador.

Efetivamente, esse catalisador dissulfeto oxazolidínico foi sintetizado em poucas etapas sintéticas seguindo o planejamento por rota combinatorial (Esquema 7).

No primeiro passo reacional introduziu-se o segundo bloco sintético pela reação do bloco sintético I, contendo a informação quiral, com diferentes aldeídos (bloco II), para levar aos ácidos tiazolidínicos correspondentes. Estes, por sua vez, foram transformados nos dissulfetos amino álcoois correspondentes por sucessivas reações 'one-pot' de redução e oxidação ao ar. Para uma primeira geração de ligantes/ catalisadores, diferentes tipos de dissulfetos oxazolilídinicos quirais foram obtidos pela reação com formaldeído (bloco III).

Utilizando essa estratégia sintética foi possível preparar diversas gerações de ligantes derivados de aminoácidos e de outros blocos sintéticos, cujas estruturas representativas ${ }^{32-37}$ de algumas classes estão mostradas na Figura 2.

$$
\underbrace{\mathrm{R}_{1}}_{\substack{1 \\ \text { Trit }}} \mathrm{OH}_{\mathrm{H}}^{\mathrm{R}_{1}}
$$

Álcool Aziridínicos ${ }^{32}$<smiles>CNC(C)C(=O)NC(C[Se]c1ccccc1)C(O)(c1ccccc1)c1ccccc1</smiles>

Seleno peptídeos ${ }^{35}$
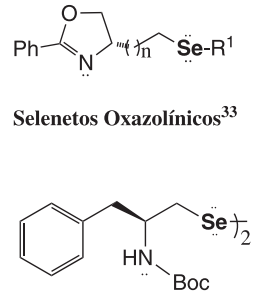

Seleno aminas ${ }^{36}$

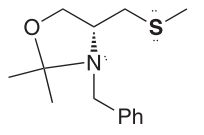

Sulfeto Oxazolidínicos ${ }^{34}$

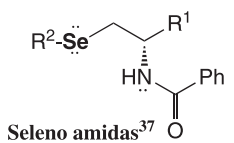

Figura 2. Ligantes/catalisadores representativos preparados no LabSelen

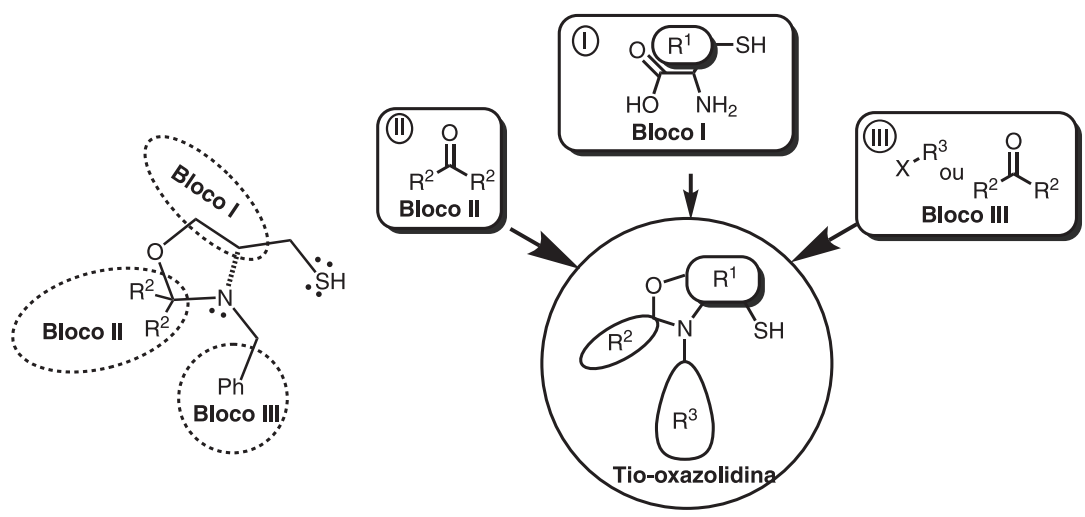




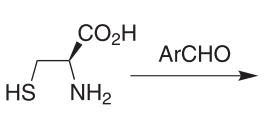

L-Cisteina

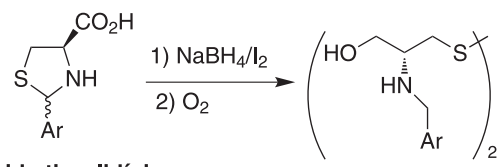

Ácido tiazolidínico
$\mathrm{CH}_{2} \mathrm{O}$

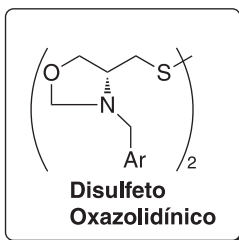

Esquema 7. Esquema sintético para a preparação do dissulfeto oxazolidínico

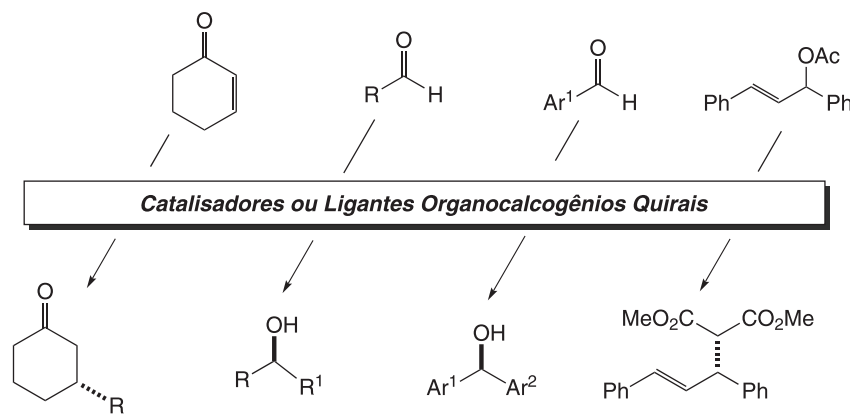

Esquema 8. Reações estereosseletivas utilizando ligantes quirais de S, Se como ligantes/catalisadores

Esses ligantes foram aplicados em diferentes reações enantiosseletivas, dentre as quais podem ser destacadas a adição 1,4 , adição de organozinco em aldeídos e substituição alílica, como mostrado no Esquema 8. ${ }^{38}$

A importância dessa estratégia sintética na busca por catalisadores privilegiados vem se mostrando altamente eficiente, e foi recentemente utilizada no desenvolvimento de novos ligantes/catalisadores promíscuos contendo selênio obtidos a partir da efedrina, ${ }^{39} \mathrm{em}$ poucas etapas sintéticas (Esquema 9).

$\mathrm{O}$ disseleneto derivado da efedrina mostrou ser um catalisador promíscuo apropriado para promover tanto a adição enantiosseletiva de organozinco em aldeídos (catalisador/ligante metálico - Esquema 10), assim como para mimetizar a enzima GPx, agindo como um potente catalisador redox, podendo ser útil em aplicação medicinal, resultando na solicitação de um depósito de patente. ${ }^{40}$ Cabe salientar ainda que os arilmetanóis enantiomericamente enriquecidos obtidos por esse processo são compostos de grande aplicabilidade em química medicinal, como será comentado mais adiante.

Recentemente uma nova abordagem para o desenvolvimento de catalisadores quirais, baseada na utilização de carboidratos $^{41}$ ou derivados de aminoácidos, tais como oxazolinas e peptídeos ${ }^{42}$ vem sendo alvo de estudos. Os (organo)catalisadores desenvolvidos vêm sendo utilizados com sucesso em diversas reações assimétricas. ${ }^{42} \mathrm{Os}$

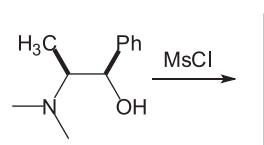<smiles></smiles>

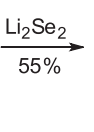<smiles>CC(C([SeH])[SnH])N(C)C</smiles><smiles>Cc1ccc(C(=O)CCCCC(=O)C(C)(C)C)cc1</smiles>

Esquema 9. Catalisadores/ligantes derivados da efedrina contendo selênio

carboidratos são uma interessante plataforma quiral para a preparação de novos compostos com potencial para o emprego como ligantes/ catalisadores em síntese assimétrica ${ }^{43}$ Eles são menos utilizados, apesar de apresentarem muitas vantagens: são facilmente disponíveis, geralmente obtidos a partir de fontes renováveis, são altamente funcionalizados e apresentam vários centros estereogênicos. Essas características permitem que uma série de ligantes sejam sintetizados e tenham seu desempenho avaliado em busca de altas seletividades para um grande número de reações enantiosseletivas. ${ }^{44}$

Aliado a isso, a versatilidade dos carboidratos confere aos ligantes deles derivados, um caráter altamente modular, permitindo o ajuste fino da estrutura do catalisador de modo a se obter informações valiosas sobre a origem da seletividade.

Nesse contexto, uma série de amino álcoois derivados dos açúcares $D$-xilose e $D$-glucosamina ( $\mathbf{L} \mathbf{1}$ e $\mathbf{L} \mathbf{2}$, respectivamente) foram sintetizados e posteriormente empregados como ligantes quirais na arilação assimétrica de aldeídos (Esquema 11). Os melhores resultados foram obtidos com o derivado xilo-furanosídico contendo um anel morfolínico em sua estrutura. Com esse ligante, uma série de diarilmetanóis foi preparada com altos rendimentos e excessos enantioméricos de até $96 \% .^{45}$

Os diarilmetanóis quirais obtidos são valiosos precursores para a síntese de compostos biológica e farmacologicamente importantes, ${ }^{46}$ tais como a orfenadrina e a neobenodina, que apresentam atividade anti-histamínica e anti-colinérgica (Esquema 12). ${ }^{47}$ Já a carbinoxamina é um antagonista histamínico $\mathrm{H}_{1}$, usado no tratamento de doenças alérgicas, assim como o cloridrato de cetirizina que é extremamente seletivo para receptores do tipo $\mathrm{H}_{1}$ e já é comercializado na sua forma enantiomericamente pura $(S)$ desde 2002. O (S)-BMS-184394 é um potente anti-carcinogênico, particularmente ativo para câncer de mama e leucemia. ${ }^{48}$

\section{Organocatálise}

Recentemente o emprego de moléculas orgânicas como mediadoras de transformações químicas uniram-se às, já bem desenvolvidas, catálise organometálica e biocatálise. A redescoberta desse conceito ocorreu por meio da divulgação dos trabalhos de Barbas III, List e MacMillan, os quais despertaram grande interesse da comunidade acadêmica. $^{49}$
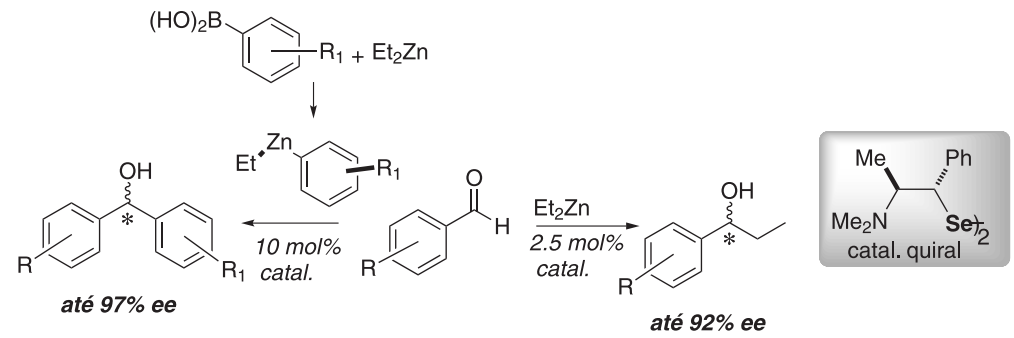

Esquema 10. Síntese e aplicação do ligante disseleneto quiral derivado da efedrina em reações de adição de organozinco em aldeídos 


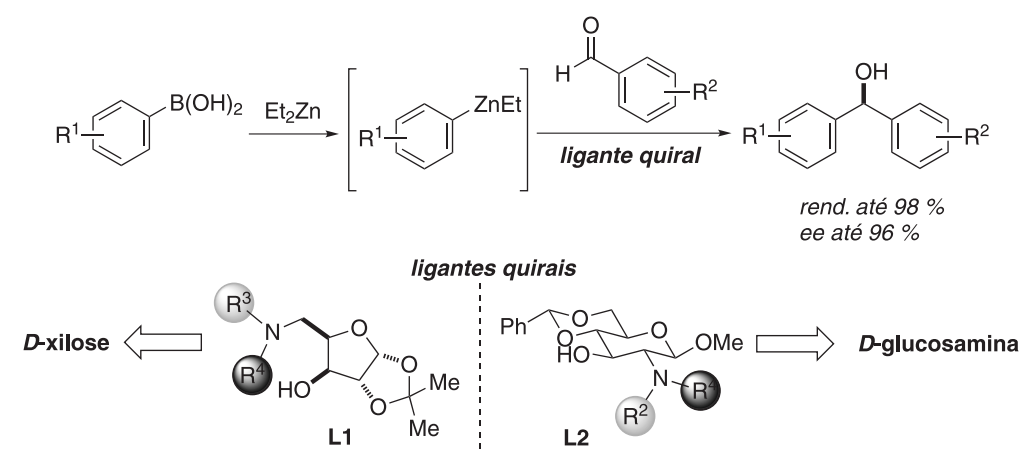

Esquema 11. Síntese e aplicação de ligantes derivados de açúcares em reações de arilação enantiosseletiva

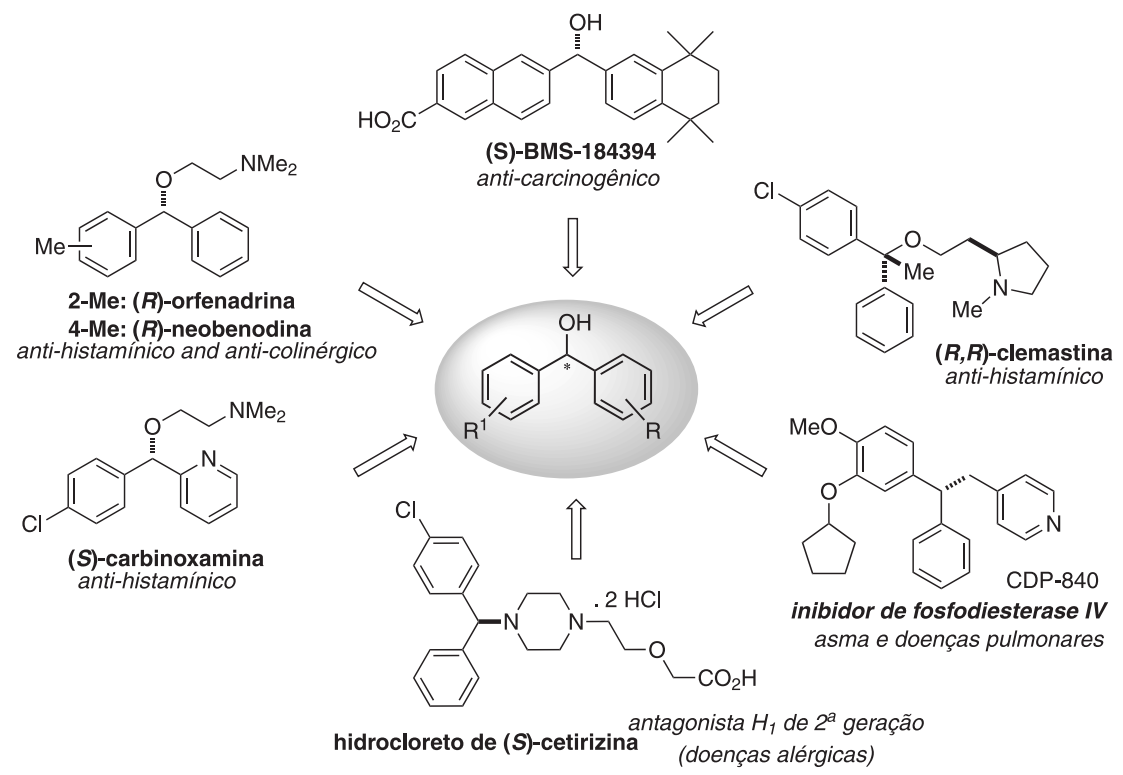

Esquema 12. Importância biológica de diarilmetanóis

Esta ferramenta tornou-se alternativa e, por vezes, complementar na utilização de catalisadores organometálicos quirais, apresentando algumas vantagens no que diz respeito a procedimentos experimentais: em geral, moléculas utilizadas como organocatalisadores são estáveis ao ar, passíveis de estocagem, baratas, de fácil obtenção e, além disso, observa-se que, em muitos casos, reações organocatalisadas não requerem a utilização de sistemas inertes, nem tampouco solventes ou reagentes anidros. ${ }^{50} \mathrm{~A}$ ausência de metais de transição configura-se também numa importante vantagem, principalmente, na síntese de produtos farmacêuticos, nos quais a contaminação por metal, mesmo que em pequenos traços, é indesejada. ${ }^{51}$

Este campo é hoje reconhecido como o terceiro pilar da catálise assimétrica, em razão do grande número de transformações e metodologias desenvolvidas utilizando organocatalisadores. ${ }^{52}$ Reações como: adição aldólica, adições conjugadas, cicloadições, $\alpha-$ e $\beta$-funcionalizações, reações de Mannich, Henry, Biginelli e Baylis-Hilmann são apenas alguns exemplos de reações que podem ser realizadas empregando esta ferramenta. ${ }^{53}$

Em uma abordagem comercial, os organocatalisadores quirais tornaram-se de grande interesse econômico, do ponto de vista industrial. ${ }^{54}$ Nesse contexto, inúmeros compostos podem ser produzidos, em nível industrial, na sua forma enantiomericamente pura, e alguns processos industriais já fazem uso da organocatálise assimétrica como mecanismo de indução de assimetria. Um exemplo pode ser observado na síntese do BMS-594726, um dos mais eficientes fármacos utilizados como inibidores da recaptação seletiva de serotonina (IRSS); desenvolvida para o tratamento de depressão, distimia, ejaculação precoce, síndrome do pânico, fobias em geral e o transtorno obsessivo compulsivo (TOC).$^{55} \mathrm{~A}$ etapa chave na síntese deste fármaco é a reação de alquilação de Friedel-Crafts, catalisada pelo organocatalisador imidazolidínico, a qual fornece o produto em um alto excesso enantiomérico, conforme pode ser observado no Esquema 13. ${ }^{56}$

Com o despontar da área, pesquisadores brasileiros começaram a se aventurar nesta nova linha e alguns trabalhos foram publicados de maneira pontual. ${ }^{57}$

Como exemplo, podemos citar a nucleação de novos grupos de pesquisas voltados ao desenvolvimento de novos métodos e modelos organocatalisados que estão se estabelecendo no Brasil, na UFSCar e UFRGS, voltados para o desenvolvimento de novos modificadores quirais pirrolidínicos, derivados da $L$-prolina e os tiazolidínicos, derivados do aminoácido natural $L$-cisteína. ${ }^{58}$ Estas duas novas classes de compostos apresentaram ótima efetividade em reações aldólicas assimétricas, fornecendo o produto em até $99 \%$ de excesso enantiomérico (Esquema 14).

Cabe ressaltar que estes compostos possuem grande possibilidade de variação estrutural, o que é bastante interessante a fim de se aperfeiçoar a estrutura desses compostos em termos de atividade e estereosseletividade, tornando-os excelentes transferidores de assimetria.

Mais recentemente, foi desenvolvido o planejamento racional de uma nova classe de organocatalisadores baseada no aminoácido $L$-Prolina e grupos hidrofóbicos, o que proporciona características especiais para aplicação em sistemas aquosos. O emprego desta metodologia está em consonância com o desenvolvimento e fortalecimento 


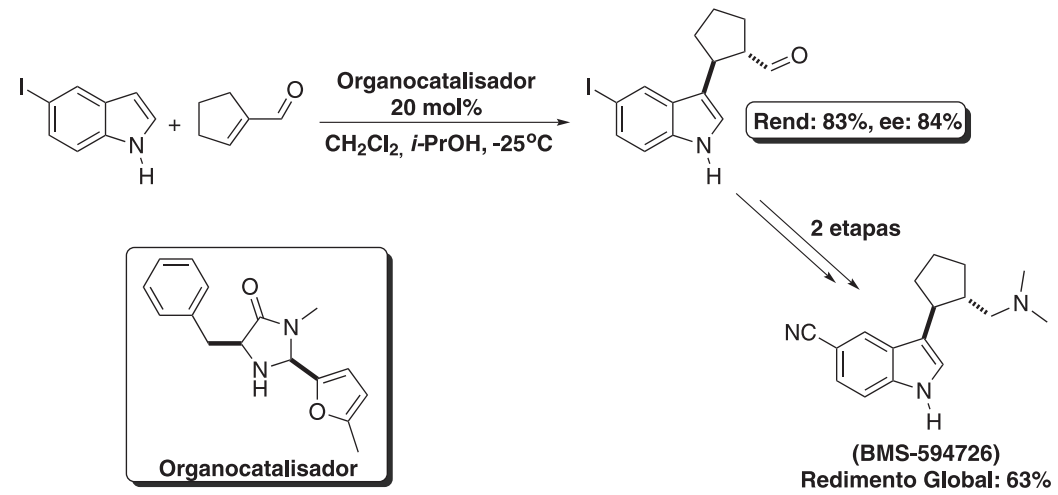

Esquema 13. Síntese do BMS-594726

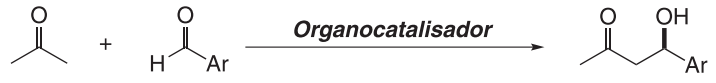

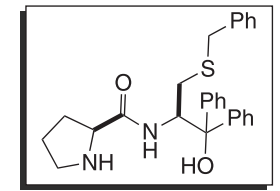

ACETONA como Solvente Rend. de até $85 \%$ ee de até $97 \%$

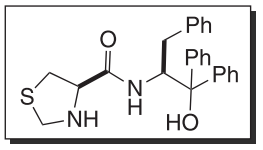

$\mathrm{NaCl}$ (sol) como Solvente Rend. de até $95 \%$ ee de até $99 \%$
Esquema 14. Organocatalisadores Prolina e Tiazolidina-Carboxamida desenvolvidos

da química verde do Brasil. Foi sintetizada uma quimioteca de novos organocatalisadores, que foram posteriormente aplicados em reações de funcionalização de aldeídos $\alpha, \beta$-insaturados, empregando solventes verdes como, por exemplo, solução saturada de $\mathrm{NaCl}$, etanol e água (Esquema 15). ${ }^{59}$

A organocatálise assimétrica pode ser considerada como uma excelente ferramenta sintética, pois agrega elegância à síntese de forma sustentável e, além dos catalisadores atuarem como agentes de transferência de quiralidade, estes possuem ótimos índices de recuperação e reutilização sem perda considerável de sua atividade catalítica. Esta metodologia deverá despertar o interesse de novos grupos, bem como, do setor produtivo na construção de blocos/ drogas quirais.

\section{CONCLUSÃO}

A maioria dos processos químicos de produção de pequenas moléculas quirais ainda não se enquadra em processos ambientalmente aceitáveis, levando a uma grande preocupação na sociedade civil, assim como das autoridades governamentais. Acordos internacionais do meio ambiente poderão levar as indústrias químicas a desenvolver processos que sejam cada vez menos prejudiciais ao meio ambiente. Assim, a preparação desses compostos por métodos mais sustentáveis deverão surgir, podendo mudar o mapa atual de produção global.

Neste contexto, a catálise assimétrica surge como uma ferramenta poderosa e ambientalmente aceitável para a síntese dessas moléculas quirais, de alto valor agregado, a partir de moléculas aquirais, utilizando um catalisador quiral. É altamente desejável que esses catalisadores sejam obtidos a partir de matérias-primas abundantes, de baixo custo e oriundas de fontes renováveis. Considerando-se esse panorama, o Brasil poderá se beneficiar dessa oportunidade, uma vez que além de possuir uma ampla biodiversidade e recursos naturais para a obtenção dos insumos para a produção de novos catalisadores quirais, vem criando competência nessa área, possuindo, atualmente, uma massa crítica considerável, tanto nessa quanto em áreas afins, que também podem contribuir de forma decisiva para o crescimento da química em nosso país.

Nesse particular cabe ressaltar que aportar recursos para o financiamento de projetos de escalonamento e produção de rotas alternativas a produtos quirais com eficácia comprovada e/ou já

Reações Sequenciais em Solventes Verdes
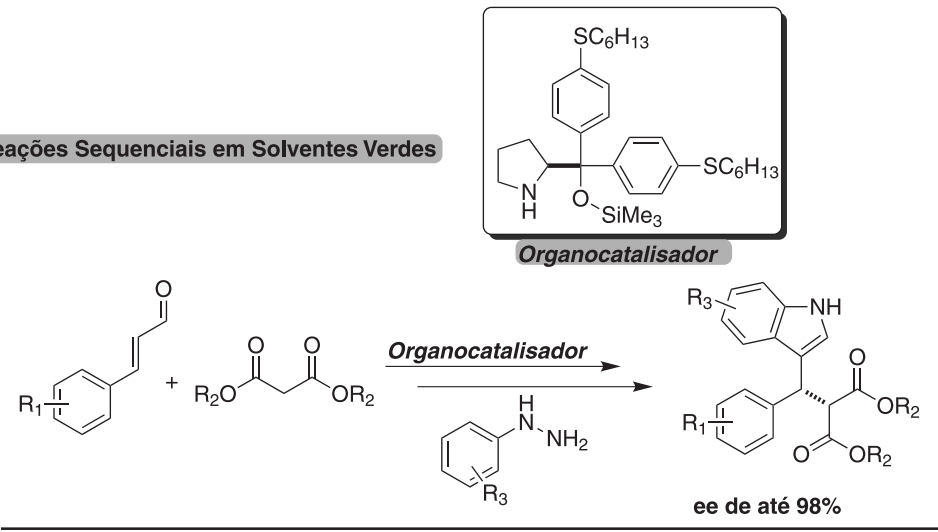

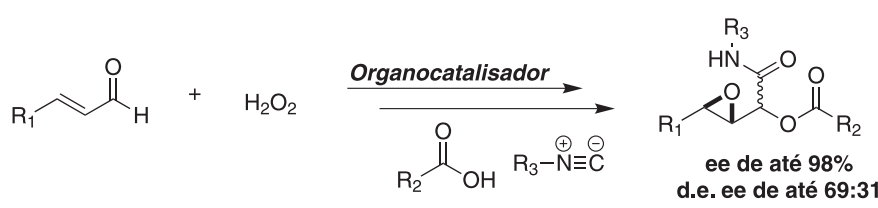

Esquema 15. Reações sequenciais organocatalisadas em solventes ambientalmente mais corretos 
existentes no mercado é meritório e necessário. Assim, financiamentos dirigidos para esses setores nos moldes do "projeto PADCT" seriam de fundamental importância para o avanço da química brasileira.

Por fim, mas não menos importante, é imperativo ressaltar que para que o Brasil assuma esse protagonismo que lhe é esperado, é necessária uma política constante de valorização da Ciência Fundamental. É a partir do fortalecimento da ciência básica que se constrói um alicerce sólido para o surgimento de soluções para os desafios científicos vindouros e crescimento econômico. Parafraseando Helmut Schwartz, Presidente da Fundação Alexander von Humboldt: "A pesquisa básica é essencial se nós queremos assegurar o futuro, porque nenhuma das questões diárias candentes podem ser resolvidas sem ela." Tradução livre de: "Fundamental research is essential if we are to secure the future because none of the burning issues of the day can be solved without it".

\section{AGRADECIMENTOS}

Às agências brasileiras de fomento à pesquisa CNPq, CAPES, FAPESC, FAPESP, FAPERGS, PRONEX, PRONEM e INCT-Catálise

\section{REFERENCIAS}

1. Pinto, A. C.; Zucco, C.; Galembeck, F.; de Andrade, J. B.; Vieira, P. C.; Quim. Nova 2012, 32, 623.

2. Asymmetric Catalysis on Industrial Scale: Challenges, Approaches and Solutions, Eds.: Blaser, H. U. and Schmidt, E. Wiley-VCH Verlag: Weinheim, 2007; Patti, A. Green Approaches To Asymmetric Catalytic Synthesis, Springer: Berlin, 2011; Luo, Z.; Zhang, S.; Chem. Soc. Rev. 2012, 41, 4736.

3. Kasprzyk-Hordern, B.; Chem. Soc. Rev. 2010, 39, 4466.

4. Rouhi, A. M.; Chem. Eng. News 2003, 81, 45.

5. http://www.chirosolve.com/docs/Technical_Presentation_7-1-2011.pdf, acessada em junho de 2013.

6. Dupont J.; Quim. Nova 2000, 23, 825; Prado, A. G. S.; Quim. Nova 2003, 26, 738; Machado, A. A. S. C.; Quim. Nova, 2011, 34, 535; Sustainable Industrial Chemistry, Eds.: Cavani, F.; Centi, G.; Perathoner, S.; Trifirò, F.; Wiley-VCH: Weinheim, 2009.

7. Dias, F. R. F.; Ferreira, V. F.; Cunha, A. C.; Revista Virtual de Química 2012, 4,840 .

8. Pilli, R. A.; Química Nova na Escola 2001, 14, 16.

9. Knowles, W. S.; Sabacky, M.; Vinineyard, B. D.; Weinkauff, D. J.; J. Am. Chem. Soc. 1975, 97, 2567.

10. Thayer, A. M.; Chem. Eng. News 2007, 85, 11; Farina, V.; Reeves, J. T.; Senanayake, C. H.; Song, J. J.; Chem. Rev. 2006, 106, 2734.

11. Valdés, R. H.; Puzer, L.; Gomes Jr., M; Marques, C. E. S. J.; Aranda, D. G. A.; Bastos, M. L.; Gemal, A. L.; Antunes, O. A. C.; Catal. Commun. 2004, 5, 631 .

12. Yoon, T. P.; Jacobsen, E. N. Science 2003, 299, 1691; Hamilton, G. L.; Kang, R. J.; Mba, M.; Toste, F. D.; Science 2007, 317, 496.

13. Schoemaker, H. E.; Mink, D.; Wubbolts, M. G. Science 2003, 299, 1694; Schmid, A.; Hollmann, F.; Park, J. B.; Buhler, B.; Curr. Opin. Biotechnol. 2002, 13, 359.

14. Bruggink, A.; Roos, E. C.; de Vroom, E.; Org. Process Res. Dev. 1998, $2,128$.

15. Brenelli, E. C. S.; Moran, P. J. S; Rodrigues, J. A. R.; Synth. Commun. 1990, 20, 261; Mantovani, S. M.; Angolini, C. F. F.; Marsaioli, A. J.; Tetrahedron: Asymmetry 2009, 20, 2635.

16. Hoffmann, I.; Silva, D. V.; Nascimento, M. G.; J. Braz. Chem. Soc. 2011, 22, 1559 .

17. Rocha, L. C.; de Souza, A. L.; Rodrigues Filho, U. P.; Campana Filho, S. P.; Sette, L. D.; Porto, A. L. M.; J. Mol. Catal. B: Enzym. 2012, 84, 160.
18. Miranda, A. S.; Miranda, L. S. M.; Souza, R. O. M. A.; Org. Biomol. Chem. 2013, 11, 3332 .

19. Machado, L. L.; Lemos, T. L. G.; Mattos, M. C.; de Oliveira, M. C. F.; Gonzalo, G.; Gotor-Fernández, V.; Gotor, V.; Tetrahedron: Asymmetry 2008, 19, 1419.

20. Araujo, D. M. F.; Vieira, G. A. B.; Mattos, M. C.; Lemos, T. L. G.; de Oliveira, M. C. F.; Melo, V. M. M.; Gonzalo, G.; Gotor-Fernández, V.; Gotor, V.; J. Mol. Catal. B: Enzym. 2009, 61, 279.

21. Barros-Filho, B. A.; Nunes, F. M.; de Oliveira, M. C. F.; Lemos, T. L. G.; Mattos, M. C.; Gonzalo, G.; Gotor-Fernández, V.; Gotor, V.; J. Mol. Catal. B: Enzym. 2010, 65, 37.

22. Missio, L. J.; Comasseto, J. V.; Tetrahedron: Asymmetry 2000, 11, 4609.

23. Barcellos, T.; Tauber, T.; Kroutil, W.; Andrade, L. H.; Tetrahedron: Asymmetry 2011, 22, 1772; Andrade, L. H.; Barcellos, T.; Org. Lett. 2009, 11, 3052.

24. Andrade, L. H.; Omori, A. T.; Porto, A. L. M.; Comasseto, J. V.; J. Mol. Catal. B: Enzym. 2004, 29, 47.

25. Brondani, P. B.; Gonzalo, G.; Fraaije, M. W.; Andrade, L. H.; Adv. Synth. Catal. 2011, 353, 2169; Brondani, P. B.; Dudek, H. M.; Reis, J. S.; Fraaije, M. W.; Andrade, L. H.; Tetrahedron: Asymmetry 2012, 23, 703.

26. Araújo, L. S.; Kagohara, E.; Garcia, T. P.; Pellizari, V. H.; Andrade, L. H.; Marine Drugs 2011, 9, 889; Silva, C. R.; Souza, J. C.; Araújo, L. S.; Kagohara, E.; Garcia, T. P.; Pelizzari, V. H.; Andrade, L. H.; J. Mol. Catal. B: Enzym. 2012, 83, 23.

27. Braga, A. L.; Appelt, H. R.; Schneider, P. H.; Silveira, C. C.; Wessjohann, L.; A. Tetrahedron: Asymmetry 1999, 10, 1733; Braga, A. L.; Lüdtke, D. S.; Wessjohann, L. A.; Paixao, M. W.; Schneider, P. H.; J. Mol. Catal. A: Chem. 2005, 229, 47.

28. Braga, A. L.; Paixão, M. W.; Godoi, M.; Dalton Trans. 2011, 40, 11347.

29. Exemplos de trabalhos pontuais desenvolvidos: Vasconcelos, M. L. A. A.; Barreiros, W. G.; Branco, M. C.; Gambardella, M. T. P.; Ribeiro, M.; Synth. Commun. 1998, 28, 4077; Ramminger, C.; Zim, D; Lando, V. R.; Fassina, V.; Monteiro, A. L.; J. Braz. Chem. Soc. 2000, 11, 105; Santos, L. S.; Fernandes, S. A.; Pilli, R. A.; Marsaioli, A. J.; Tetrahedron: Asymmetry 2003, 14, 2515.

30. Correia, C. R. D.; Oliveira, C. C.; Salles Jr, A. G.; Santos, E. A. F.; Tetrahedron Lett. 2012, 53, 3325; Oliveira, C. C.; Angnes, R. A.; Correia, C. R. D.; J. Org. Chem. 2013, 78, 4373.

31. Yamakawa, M.; Noyori, R.; J. Am. Chem. Soc. 1995, 117, 6327.

32. Braga, A. L.; Paixão, M. W.; Westermann, B.; Schneider, P. H.; Wessjohann, L. A.; J. Org. Chem. 2008, 73, 2879.

33. Braga, A. L.; Lüdtke, D. S.; Alberto, E. E.; J. Braz. Chem. Soc. 2006, $17,11$.

34. Schneider, P. H.; Schnrekker, H. S.;Silveira, C. C; Wessjohann, L. A.; Braga, A. L.; Eur. J. Org. Chem. 2004, 2715.

35. Schwab, R. S.; Soares, L. C.; Dornelles, L.; Rodrigues, O. E. D.; Paixão, M. W.; Godoi, M.; Braga, A. L.; Eur. J. Org. Chem. 2010, 3574

36. Braga, A. L.; Paixão, M. W.; Lüdtke, D. S.; Silveira, C. C.; Rodrigues, O. E. D.; Org. Lett. 2003, 5, 2635.

37. Braga, A. L.; Vargas, F. Sehnem, J. A.; Braga, R. C.; J. Org. Chem. 2005, 70, 9021.

38. Braga, A. L.; Lüdtke, D. S.; Vargas, F.; Curr. Org. Chem. 2006, 10, 1921; Braga, A. L.; Lüdtke, D. S.; Vargas, F.; Braga, R. C.; Synlett 2006, 1453.

39. Soares, L. C.; Alberto, E. E.; Schwab, R. S.; Taube, P. S.; Nascimento, V.; Rodrigues, O. E. D.; Braga, A. L.; Org. Biomol. Chem. 2012, 10, 6595.

40. Braga, A. L..; Botteselle, G. V.; Bettanin, L.; Rodrigues, O. E. D.; Soares, L. C.; Br 102012 0324474, 2012.

41. Wouters, A. D.; Lüdtke, D. S.; Org. Lett. 2012, 14, 3962; Wouters, A. D.; Bessa, A. B.; Sachini, M.; Wessjohann, L. A.; Lüdtke, D. S.; Synthesis 2013, 45, 2222. 
42. Schwab, R. S.; Schneider, P. H.; Tetrahedron 2012, 68, 10449.

43. Ferreira, V. F.; da Rocha, D. F.; da Silva, F. C.; Quim. Nova 2009, 32, 623.

44. Para revisões sobre ligantes derivados de carboidratos em catálise assimétrica: Diéguez, M.; Pàmies, O.; Claver, C.; Chem. Rev. 2004, 104 3189; Lehnert, T.; Ozuduru, G.; Grugel, H.; Albrecht, F.; Telligmann, S. M.; Boysen M. M. K.; Synthesis 2011, 2685.

45. Wouters, A. D.; Trossini, G. H. G.; Stefani, H. A.; Lüdtke, D. S.; Eur. J. Org. Chem. 2010, 2351.

46. Paixão, M. W.; Braga, A. L.; Lüdtke, D. S.; J. Braz. Chem. Soc. 2008, 19, 813; Schmidt, F.; Stemmler, R. T.; Rudolph, J.; Bolm, C.; Chem. Soc. Rev. 2006, 35, 454.

47. Rekker, R. F.; Timmerman, H.; Harms, A. F.; Nauta, W. T.; Arzneim.-Forsch. 1971, 21, 688-691.

48. Klaholz, B. P.; Mitschler, A.; Moras, D.; J. Mol. Biol. 2000, 302, 155.

49. List, B.; Lerner, R. A.; Barbas, C. F.; J. Am. Chem. Soc. 2000, 122, 2395; Ahrendt, K. A.; Borths, C. J.; MacMillan, D. W. C.; J. Am. Chem. Soc. 2000, 122, 4243 .

50. Wende, R. C.; Schreiner, P. R.; Green Chem. 2012, 14, 1821.

51. Garrette, C. F.; Prasad, K.; Adv. Synth. Catal. 2004, 346, 889.

52. Amarante, G. W.; Coelho, F.; Quim. Nova 2009, 32, 469; Nielsen, M.; Worgull, D.; Zweifel, T.; Gschwend, B.; Bertelsen, S.; Jorgensen, K.; Chem. Commun. 2011, 47, 632.
53. Enders, D.; Grondal, C.; Hüttl, M. R. M.; Angew. Chem. Int. Ed. 2007, 46, 1570; MacMillan, D. W. C.; Nature 2008, 455, 304; Bertelsen, S.; Jørgensen, K. A.; Chem. Soc. Rev. 2009, 38, 2178; Narayanaperumal, S.; Rivera, D. G.; da Silva, R. C.; Paixão, M. W.; ChemCatChem. 2013, doi:10.1002/cctc.201200936.

54. Asymmetric Catalysis on Industrial Scale, Challenges, Approaches and Solutions, Blaser, H. -U.; Schmidt, E., Eds.; Wiley/VCH, Weinheim, 2004.

55. Spinks, D.; Spinks, G.; Curr. Med. Chem. 2002, 9, 799.

56. King, H. D.; Meng, Z.; Denhart, D.; Mattson, R.; Kimura, R.; Wu, D.; Gao, Q.; Macor, J. E.; Org. Lett. 2005, 7, 3437.

57. Angelice, C.; Corrêa, R. J.; Garde, S. J.; Tomasini, C.; Tetrahedron Lett. 2009, 50, 814; Martins, E. F.; Pliego, J. R.; ACS Catal. 2013, 6, 613.

58. Schwab, R. S.; Galetto, F. Z.; Azeredo, J. B.; Braga, A. L.; Lüdtke, D. S.; Paixão, M. W.; Tetrahedron Lett. 2008, 49, 5094; Rambo, R. S.; Schneider, P. H.; Tetrahedron: Asymmetry 2010, 21, 2254.

59. Deobald, A. M.; Corrêa, A. G.; Rivera, D. G.; Paixão, M. W.; Org. Biomol. Chem. 2012, 10, 768; Feu, K. S.; Deobald, A. M.; Narayanaperumal, S.; Corrêa, A. G.; Paixão, M. W.; Eur. J. Org. Chem. 2013, DOI: $10.1002 /$ joc. 201300431

60. Kneiß1, D.; Schwarz, H.; Angew. Chem. Int. Ed. 2011, 50, 12370. 